\title{
Algunas reflexiones sobre la enseñanza de la Ley de Boyle desde la perspectiva molar y molecular propuesta por Jensen utilizando la resolución de problemas
}

\author{
Some reflections on the teaching of the Loyle's Law from the perspective molar and \\ molecular proposed by Jensen using the troubleshooting.
}

Javier Mauricio Rache, Miguel Ángel Álvarez Penagos y Quira Alejandra Sanabria

Universidad Pedagógica Nacional. chemisatan@yahoo.com, mangel.85@hotmail.com, qsanabria@gmail.com

\section{Resumen}

La visión tan reducida de la ciencias, en la que se ve a ella como un cúmulo de conocimientos, finalizada y llena de verdades universales irrefutables, puede ser modificada a través de una propuesta educativa apoyada en la perspectiva elaborada por Jensen (1998), atendiendo a los niveles molar y molecular que defiende en sus publicaciones, y considerando la metodología de resolución de situaciones problemáticas (conceptuales y contextualizadas) para incentivar la creatividad de los estudiantes y su pensamiento divergente (García,2003). Se espera que en estas condiciones los estudiantes desarrollen las habilidades científicas, cognitivas y actitudinales requeridas para explorar fenómenos naturales con la profundidad que se merecen, posibilitándoles condiciones óptimas para la consecución de los niveles de aprendizaje propuestos en los estándares de calidad en ciencias naturales para la educación media.

Esto se puede llevar a cabo, como es lógico, con aualquier núdeo temático de Química. La presente propuesta se circunscribe en la temática del estado de agregación gas, y en especial en el estudio de la ley de Boyle, de las variables que intervienen en ella, en la teoría cinético-molecular y en el modelo molecular daltoniano que la sustenta.

\section{Palabras clave}

Gases, enseñanza de las ciencias, resolución de problemas, nivel molar y nivel molecular.

\section{Abstract}

The vision so reduced of the sciences, which sees it as a heap of knowledge, conduded and full of irrefutable universal truths, can be changed through an educational proposal based on the perspective developed by Jensen (1998), assisting at the molar and molecular levels that defends in his publications, and considering the methodology of resolution of problematic situations (conceptual and contextualised) to stimulate student's creativity and their divergent thought (García, 2003). It is expected that under these conditions the students develop the scientific skills, cognitive and attitudes required to explore natural phenomena with the depth they deserve, facilitating conditions for achieving optimum levels of learning proposed in the standards of quality in natural science for the half education.

This can be done, of course, with any thematic nudeus of Chemistry. The proposed present is bounded in the thematic of the state of aggregation gas, and especially in the study of the Boyle's 
law of the variables involved in it, in the kinetic-moleaular theory and molecular Daltonian model that supports it.

Keywords

Gases, science education, problem solving, molar and molecular level.

\section{Introduccion}

La enseñanza de los gases en la educación media es trabajada de una manera bastante superficial, ya que se basa principalmente en el desarrollo de ejercicios de lápiz y papel, que parten simplemente de las fórmulas derivadas de sus diferentes leyes, sin concebir que el estudio cualitativo de las propiedades de las mismas, es el aspecto de mayor relevancia; por supuesto, auando los maestros intentan introducir la resolución de problemas en su actividad educativa, utilizan "problemas" de tipo cuantitativo para enseñar y evaluar los conocimientos, debido probablemente a que suponen que su solución correcta indica que el alumno domina el contenido, tanto en información como en comprensión (Balocchi y otros 2004).

Sin embargo, algunas investigaciones Chiu (2001) citado por Balocchi y otros (2004); apuntan a que los estudiantes son capaces de resolver problemas usando algonitmos sin necesidad de comprender los conceptos químicos implícitos en las preguntas, informando muchas veces la respuesta esperada pero ayo significado ignoran. Es así como Nurrenbern (1987) citado por Balocchi y otros (2004); al realizar un estudio sobre estequiometria y leyes de los gases, encontró que el número de alumnos que podía resolver problemas tradicionales era mucho mayor que los que podían responder correctamente dichas preguntas cuando eran transformadas en preguntas conceptuales. Este trabajo mostró, por ejemplo, que cerca de dos tercios de los estudiantes no entendieron la propiedad de un gas de ocupar todo el volumen del reaipiente que los contiene, sin embargo, eran capaces de citar el hecho de que los gases tienen un volumen indefinido.

Lo anterior ha conducido a que las dases de química se transformen en aburridas sesiones de matemáticas, porque en ellas se dedica la mayor parte del tiempo al simple reemplazo de fórmulas para llegar a la única solución posible a dichos ejercicios; en este proceso se disminuye la calidad educativa de las dases de ciencias, pues no se logra llegar al cometido planteado por los estándares de calidad del ministerio de educación nacional (2004).

Si objetivo de la ciencia es el de generar conocimiento, contribuir a la formación de ciudadanos y ciudadanas capaces de razonar, debatir, producir, convivir y desarrollar al máximo su potencial creativo (lineamientos curriculares MEN 2004), pero a la vez, y basados en lo expuesto anteriormente observamos que la enseñanza de la misma se enfoca únicamente a repetir sucesos sin significado alguno para el estudiante, donde al parecer este último es un agente pasivo; entonces por mas que se hable que el sistema educativo tradicional es ahora constructivista se sigue cayendo en las mismas falencias (ciencia descontextualizada, bajos niveles de comprensión, actitud aprehensiva hacia la ciencia y hacia su estudio, apatía por el conocimiento científico y por los procesos de investigación, etc.), entonces se hace necesario abordar el estudio de la disciplina desde otra perspectiva didáctica.

Por ello se plantea en la presente propuesta el estudio de una temática espećfica, abordándola desde una estructura lógica de la disciplina basada en su historia y su epistemología (Jensen, 1998), mediante la resolución de problemas aualitativos y contextualizados (Garća, 2003); para lograr que los estudiantes sean capaces de formular preguntas, de sugerir soluciones espeáficas sobre una observación, sobre una experiencia o sobre las aplicaciones de teorías científicas, además de formular hipótesis, con base en el conocimiento cotidiano, teorías y modelos científicos MEN (2004). 


\section{Consideraciones preliminares}

Atendiendo a la parte disciplinar se analizará el estudio de los gases en un primer nivel desde el mundo macroscópico o molar seguidamente del nivel molecular (Jensen 1998). Ello está de acuerdo con el hecho de que un fenómeno puede ser estudiado y explicado desde estos dos primeros niveles de profundidad, dependiendo, daro está, de los intereses de los estudiantes y del nivel educativo en que se encuentren, en este caso básica secundaria.

En primera medida es necesaria la organización de los diferentes conceptos involucrados, desde la visión molar, en este caso en particular se formulará el análisis de las propiedades de los gases y sus variables (presión, temperatura, volumen y cantidad), para plantear, desde allí, las diferentes leyes que explican su comportamiento.

La teoría cinético-molecular de los gases y el modelo daltoniano que conlleva a la explicación de los fenómenos de los gases a nivel molecular, serian estudiados en un segundo momento.

Ahora, teniendo dara estas dos visiones de un mismo fenómeno (estados de los gases), se ve necesaria la implementación de la resolución de problemas en el aula de clase, para darle la oportunidad al estudiante de utilizar ese conocimiento construido al proporcionarle una aplicabilidad, dicho conocimiento servirá como punto de partida para la generación de múltiples ideas a través del pensamiento divergente desarrollado en este proceso por parte del estudiante y desde allí poder darle solución a los diferentes problemas cotidianos, el cual es otro de los propósitos de los estándares de calidad para educación media.

Los procesos histórico y epistemológico de la Química se hacen de vital importancia para la enseñanza de esta temática, porque dan cuenta de un desarrollo paulatino de la ciencia y de sus modelos (Garcáa 2003), donde hay cabida para los errores y para el aprendizaje desde ellos; ubicando a los científicos en un contexto social, político, económico y tecnológico que reperaute innegablemente en sus proyectos investigativos, entendiendo la ciencia como un constructo social e individual (Lakatos 1971); interpretando mejor las razones que motivaron al estudio o a la propuesta de teorías o modelos; comprendiendo en mayor medida el proceso de investigación y la posterior construcción del conocimiento científico y de los modelos que lo sustentan.

Por otro lado evidenciar que el estudio la Química se basa en la interpretación de modelos acordes con los fenómenos estudiados y no en la realidad misma (Caldin 2002), transforma el proceso enseñanza aprendizaje de la química en algo más interesante porque el estudiante observa que el conocimiento científico no es una realidad absoluta, que existen constantes avances en los modelos interpretativos y que estos son usados de aarerdo a la necesidad de los investigadores y la interpretación que hacen de la realidad y los fenómenos que estudian. (Gire 1992) (Lombardi 1998)

\section{Desarrollo}

En primera medida y antes de la intervención con la propuesta se hace necesario brindar, a los educandos, un heurístico general para la solución de problemas, con el fin de guiar el proceso de resolución al que se enfrentarán posteriormente.

El siguiente paso en la presente propuesta es el diseño de situaciones problémicas contextualizadas, ya que estas le dan más valor y significado a los conceptos que se van construyendo en la mente de los estudiantes (Garća 2003), en la primera fase se plantearán problemas relacionados con los conceptos básicos de volumen, presión y temperatura, los cuales se 
relacionan con las propiedades físicas de los gases, respondiendo a su vez al nivel molar propuesto por Jensen.

En un segundo momento se tratarán las situaciones problémicas concemientes a la teoría cinético-molecular y al modelo daltoniano de las interacciones moleculares, ya que es este modelo el que explica mejor el comportamiento de los gases, esto corresponde al nivel molecular propuesto por Jensen. Es muy importante resaltar que esta última parte es la más importante para la verdadera comprensión de los fenómenos que atañen al comportamiento de los gases.

Por último se presentarán las situaciones problemáticas relativas a la ley de Boyle, que es la temática sobre la cual se plantea la presente propuesta.

\section{Apreciaciones finales}

Para cumplir con lo establecido por el MEN en los lineamientos auriaulares, se hace trascendental un estudio histórico y epistemológico profundo de la Química, ya que de esta manera se pueden direccional mejor los procesos educativos.

La resolución de problemas cumple una múltiple finalidad, en el sentido que ayuda a los profesores a innovar sus prácticas docentes; ayuda a los jóvenes a interpretar y analizar mejor los conocimientos construidos, y a encontrarle un sentido práctico y contextualizado a los conceptos desarrollados; fortalece las actitudes positivas hacia el estudio de las ciencias y la investigación cientúfica y por último ayuda también a desarrollar algunas habilidades de pensamiento.

\section{Bibliografia}

Balocchi Emilio, Brenda Modak, Raúl Cerón y Juan Guerrero. (2004). Estudio sobre la resolución de problemas conceptuales y comprensión de conceptos en química . Revista Chilena de Educación Científica; 3, [2].

Caldin, E. (2002). The structure of chemistry in relation to the philosophy of science. Hyle Internacional Joumal for the Philosophy of Chemistry. (2002).

García García José Joaquín. (2003). Didáctica de las ciencias resolución de problemas y desarrollo de la creatividad .Cooperativa Editorial Magisterio. Bogotá. (2003).

García Garcáa José Joaquín. (2000).La solución de situaciones problemáticas: Una estrategia didáctica para la enseñanza de la química. Enseñanza De Las Ciencias. Vol 18 \#1 Universidad De Antioquia Medellín.

Gire, R. N. (1998). La explicación de la ciencia. Un acercamiento cognoscitivo. Colección Ciencia Básica. México. Consejo Nacional De Ciencia Y Tecnología. (1992).

Jensen, W. B. (1998). Logic, history and the chemistry textbooks. Joumal of Chemical Education. Vol 75. \# 6, 7 Y 8 .

Lakatos, I (1971). La metodología de los programas de investigación científica.

Lombardi, O. (1998). La noción de modelo en ciencias. Revista Educación en Ciencias. Vol. 2 Num.4.

Ministerio De Educación Nacional. (2004).Estándares básicos de competencias en ciencias naturales y ciencias sociales.

Ministerio de Educación Nacional (2004).Serie lineamientos aumiaulares. 